\title{
Torsion pendula with electromagnetic drive and detection system for measuring the complex shear modulus of liquids in the frequency range $80-2500 \mathrm{~Hz}$
}

\author{
C. Blom and J. Mellema
}

Rheology Group, Department of Applied Physics, Twente University of Technology, Enschede (The Netherlands)

\begin{tabular}{ll} 
Notation & \\
$a$ & coil height \\
$A$ & apparatus constant \\
$B$ & magnetic induction \\
$C_{1}, C_{2}$ & apparatus constants \\
$d$ & diameter torsion rod \\
$D$ & pendulum damping \\
$E$ & apparatus constant \\
$F_{0}$ & top frequency \\
$G$ & shear modulus torsion rod \\
$G^{*}=G^{\prime}+i G^{\prime \prime}$ & complex shear modulus \\
$h$ & length torsion rod \\
$H$ & transfer function \\
$i$ & $\sqrt{-1}$ \\
$I$ & moment of inertia \\
$J_{0}$ & excitation-current amplitude \\
$J_{\text {exc }}$ & excitation current \\
$K$ & torsion spring constant \\
$l$ & length pendulum mass \\
$M$ & torque \\
$n$ & number of coil turns \\
$p$ & dipole moment \\
$Q=\omega_{0} / \Delta \omega$ & mechanical quality \\
$r$ & radius pendulum mass \\
$R$ & Re $\{Z\}$ \\
$t$ & time \\
$T$ & temperature \\
$U$ & induction voltage \\
$U_{0}$ & induction-voltage amplitude \\
$x$ & distance \\
$X$ & Im $\{Z\}$ \\
$Z=R+i X$ & liquid impedance \\
924 & \\
& \\
\hline &
\end{tabular}

Abstract: An apparatus for the measurement of liquid complex shear viscosity in the frequency range $80-2500 \mathrm{~Hz}$, with the use of a torsion pendulum operating in forced oscillation, is described. The drive and detection system consists of a magnet inside the pendulum, two excitation and two measuring coils. The determination of the complex shear viscosity is based on the measurement of the resonance frequency and the damping of the torsion pendulum.

The feasibility of this method is demonstrated with a number of Newtonian liquids in the viscosity range 0.3 to $60 \mathrm{~m} \mathrm{~Pa}$. Results for a viscoelastic polymer solution are presented. A comparison is made with other apparatus working in the same frequency range.

Key words: Torsion pendulum instrument, complex shear modulus, viscoelastic liquid, audio-frequency

$\begin{array}{ll}Z_{\mathrm{cyl}} & \begin{array}{l}\text { characteristic cylindrical impedance } \\ Z_{\mathrm{pl}}\end{array} \\ \alpha & \text { characteristic plane impedance } \\ \alpha_{M} & \begin{array}{l}\text { angle } \\ \text { coefficient of linear expansion of the } \\ \text { pendulum mass }\end{array} \\ \alpha_{R} & \text { coefficient of linear expansion of the torsion } \\ & \text { rod } \\ \dot{\gamma} & \text { rate of shear } \\ \delta & \text { penetration depth } \\ \eta & \text { steady-state viscosity } \\ \eta_{S} & \text { solvent viscosity } \\ \theta & \text { angular displacement } \\ \theta_{0} & \text { angular-displacement amplitude } \\ \mu_{0} & =4 \pi \cdot 10^{-7} \text { Vs/Am } \\ \varrho & \text { density } \\ \varphi & \text { phase angle } \\ \omega & \text { angular frequency } \\ \omega_{0} & \text { top angular frequency } \\ \Delta \omega & \text { band-width }\end{array}$

\section{Introduction}

The linear viscoelastic behaviour of liquid systems in a wide frequency range is a growing field of interest. Systems investigated are amongst others polymer solutions [1], microemulsions [2], ordered latices [3], blood and synovial fluids [4]. In the high-frequency range $(10-200 \mathrm{kHz})$ a number of instruments based on quartz crystal and nickel tube resonators are used [5]. In the low-frequency range (less than $10 \mathrm{~Hz}$ ) a lot of 
different types of dynamic viscometers are used [1]. In the intermediate range the choice of instruments described in the literature $[3,6,7,8]$ is limited. The information, in particular on design aspects, about developed instruments is scant, with the lumped resonator of Schrag and Johnson $[6,9]$ as an exception. Their apparatus, however, is rather temperature-dependent and has a relatively complicated detection system. In this paper a new torsion pendulum is presented which does not have these disadvantages. The operating principles are described in Section 2. To make copying of the apparatus more easy, in Section 3 the design aspects are elucidated. Its feasibility is demonstrated in Sections 4 and 5. The maximum rate of shear can be deduced from measurements of the angular amplitude of the pendulum and the shear wave propagation in the surrounding liquid. This subject is treated in Section 6. Finally, the characteristics of the new torsion pendulum are compared with apparatus described in the literature.

\section{Measuring principle}

A simplified diagram of the torsion pendulum is given in figure 1 . The torsional vibrations are excited and measured by means of coils, which are positioned just outside the sample holder.

The motion of the pendulum in forced vibration is described by

$$
I \ddot{\theta}+(Z+D) \dot{\theta}+K \theta=M,
$$

where $\theta$ is the angular displacement and $I$ is the momentum of inertia of the cylindrical mass. The moment of inertia of the torsion rod is neglected. The damping $Z=R+i X$ is due to the surrounding liquid; $D$ is the damping of the torsion pendulum itself, $K$ is

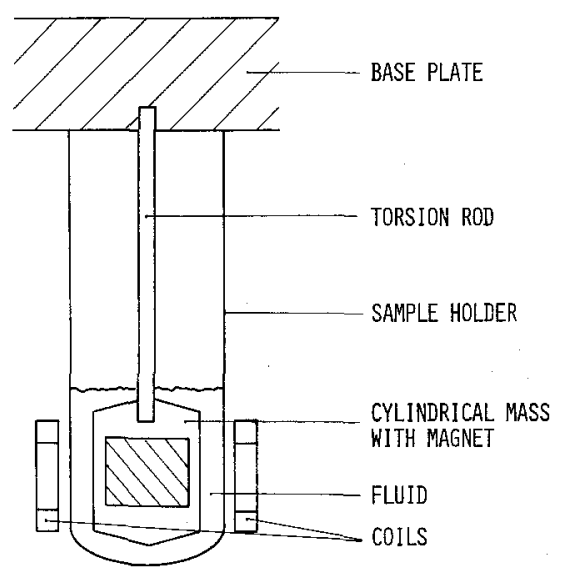

Fig. 1. Simplified geometry of the instrument. the torsion-spring constant and $M$ is the external torque exerted on the pendulum.

For the small angular displacements used the torque is proportional to the harmonically oscillating current through the excitation coils:

$$
M=C_{1} J_{e x}=C_{1} J_{0} e^{i \omega t} \text {. }
$$

The direction of the magnetic dipole of the magnet with respect to the measuring coils is chosen such that for the small angular displacements used the induction voltage $U$ is proportional to the angular velocity:

$$
U=C_{2} \dot{\theta} .
$$

Differentiating (1) with respect to time and substituting (2) and (3) into it results in

$$
I \ddot{U}+(R+i X+D) \dot{U}+K U=i \omega C_{1} C_{2} J_{0} e^{i \omega t} .
$$

A solution for the induction voltage is

$$
U=U_{0} e^{i(\omega t+\varphi)} \text {, }
$$

where $\varphi$ is the phase difference between the induction voltage and the excitation current. From (4) and (5) the modulus of the transfer function $H$ is calculated:

$$
|H|=\frac{U_{0}}{J_{0}}=\frac{\omega C_{1} C_{2}}{\left\{\left(-I \omega^{2}+K-X \omega\right)^{2}+(D \omega+R \omega)^{2}\right\}^{1 / 2}} .
$$

The frequency for which this function has a maximum is in good approximation $\left(X^{2} \ll K I\right)$ given by

$$
\omega_{0}=\frac{-X}{2 I}+\sqrt{\frac{K}{I}} .
$$

The band-width, defined as the difference between the frequencies for which the value of the transfer function is $\frac{1}{2} \sqrt{2}$ times its maximum, is in good approximation $\left((D+R)^{2} \ll K I\right)$ given by

$$
\Delta \omega=\frac{(D+R)}{I} .
$$

The error in this approximation is at most $0.3 \%$ for the highest viscosity $(58 \mathrm{~m} \mathrm{Pas})$ and the lowest frequency $(80 \mathrm{~Hz})$ used.

From (7) and (8) it follows that from a measurement in vacuum $(R=X=0)$ and a measurement in liquid the $R$ and $X$ of the liquid are determined by:

$$
\begin{aligned}
& R=I\left(\Delta \omega_{\mathrm{liq}}-\Delta \omega_{\mathrm{vac}}\right), \\
& X=2 I\left(\omega_{0_{\mathrm{vac}}}-\omega_{0_{\mathrm{liq}}}\right)
\end{aligned}
$$

The damping due to the surrounding liquid (identical to liquid impedance) is composed of two parts:

$$
Z=2 \pi r^{3} 1 Z_{\mathrm{cyl}}+\frac{\pi r^{4}}{\cos \alpha} Z_{\mathrm{pl}}
$$


The first term is due to the shear waves with a cylindrical wave front from the side of the pendulum. The second term is due to plane-shear waves from the top and bottom of the pendulum. The cylindrical part has a radius $r, l$ is its length and $\alpha$ is the small angle that top and bottoms surface make with a plane perpendicular to the pendulum axis. The edge effects and the influence of that part of the torsion rod which is surrounded by the liquid are neglected.

Schrag et al. [6] gave a first-order approximation for the characteristic cylindrical-shear impedance:

$$
Z_{\mathrm{cyl}}=Z_{\mathrm{pl}}-\frac{3 i}{2 \varrho \omega r} Z_{\mathrm{pl}}^{2} .
$$

The relation between the components of the complexshear modulus $G^{*}=G^{\prime}+i G^{\prime \prime}$ and the $R_{\mathrm{pl}}$ and $X_{\mathrm{pl}}$ are given by

$$
\begin{aligned}
& G^{\prime}=\left(R_{\mathrm{p} 1}^{2}-X_{\mathrm{p} 1}^{2}\right) / \varrho, \\
& G^{\prime \prime}=2 R_{\mathrm{pl}} X_{\mathrm{pl}} / \varrho .
\end{aligned}
$$

So when (9) through (14) is used, the real and imaginary part of the characteristic-shear impedance are:

$$
\begin{aligned}
& R_{\mathrm{pl}}=\frac{1}{2} A\left(\Delta \omega_{\mathrm{liq}}-\Delta \omega_{\mathrm{vac}}\right)-E G^{\prime \prime} / \omega, \\
& X_{\mathrm{pl}}=A\left(\omega_{0_{\mathrm{vac}}}-\omega_{0_{\mathrm{liq}}}\right)+E G^{\prime} / \omega,
\end{aligned}
$$

where the apparatus constants $A$ and $E$ are given by

$$
\begin{aligned}
& A=2 I /\left(2 \pi r^{3} l+\pi r^{4} / \cos \alpha\right), \\
& E=3 l /\left(2 r l+r^{2} / \cos \alpha\right) .
\end{aligned}
$$

The apparatus constants $A$ and $E$ are determined by calibration with Newtonian liquids, for which $R_{\mathrm{pl}}=$ $X_{\mathrm{pl}}=\sqrt{\varrho \eta \omega / 2} . R_{\mathrm{pl}}$ and $X_{\mathrm{pl}}$ are calculated from (15) and (16) with help of an iteration procedure starting with $G^{\prime}=G^{\prime \prime}=0$.

\section{Apparatus}

\subsection{General arrangement and data handling}

As is shown in figure 2 the magnet is situated between the excitation and the measuring coil. The torsional vibrations are excited by means of an alternating magnetic field produced by the excitation coil. The current through the coil is generated by the frequency synthesizer (HP $3325 \mathrm{~A}$ ). The frequency and current settings are controlled by the microprocessor (HP $9825 \mathrm{~A}$ ).

The torsional amplitude is measured with help of the measuring coil. The signal from this coil is amplified, band-pass filtered, and fed into a voltmeter

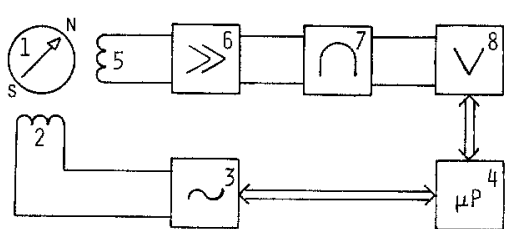

Fig. 2. General arrangement. 1. magnet (top view); 2. excitation coil; 3 . frequency synthesizer; 4 . microprocessor; 5 . measuring coil; 6 . amplifier; 7 . band-pass filter; 8 . voltmeter.

(HP 3455 A), which is controlled by the microprocessor. By measuring a number of points of the resonance curve, the resonance frequency and the band-width are calculated. The data handling is the same as used by Oosterbroek et al. [5]. The time needed for one measurement is about one minute.

\subsection{Mechanical design}

The mechanical details of the torsion pendulum are shown in figure 3 . Four different pendula have been used. The dimensions and calculated resonance frequencies are given in table 1 .

The main feature of the design is the temperature-independent resonance frequency. This is achieved by using a special nickel alloy for the torsion rod. The material used is Thermelast 4290, manufacturer Vakuumschmelze $\mathrm{GmbH}$, Hanau, Germany [10]. The resonance frequency of the pendulum in vacuum is given by

$$
\omega_{0}=\sqrt{\frac{K}{I}}
$$

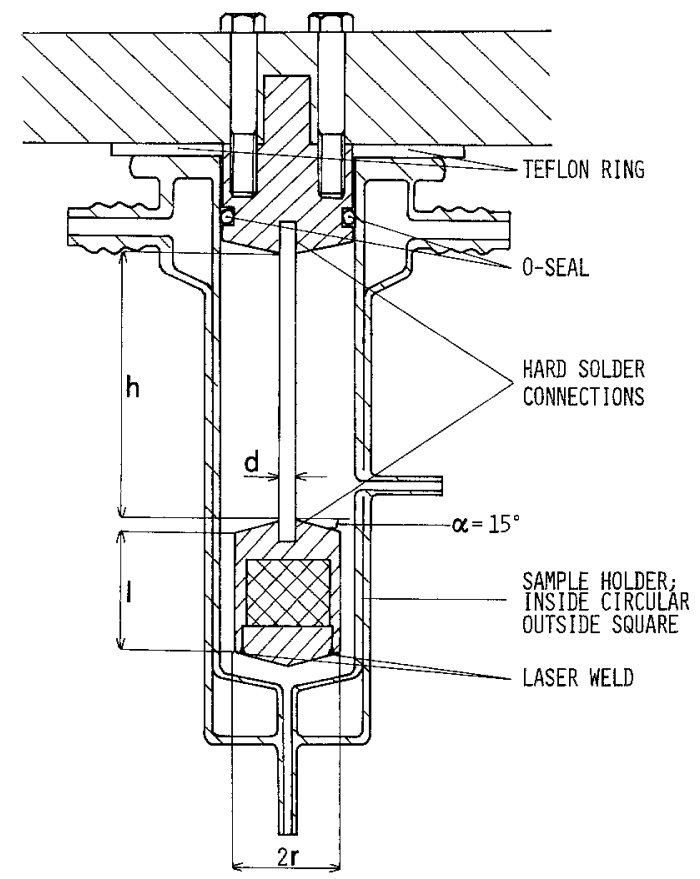

Fig. 3. Mechanical design. 
Table 1. Pendulum dimensions

\begin{tabular}{llllllllr}
\hline $\begin{array}{l}\text { pen- } \\
\text { dulum } \\
\text { number }\end{array}$ & $\begin{array}{l}d \\
\text { num] }]\end{array}$ & $\begin{array}{l}h \\
{[\mathrm{~mm}]}\end{array}$ & $\begin{array}{l}l \\
{[\mathrm{~mm}]}\end{array}$ & $\begin{array}{l}I \\
{[\mathrm{~mm}]}\end{array}$ & $\begin{array}{l}F_{0} \\
{\left[10^{-7}\right.} \\
\left.\mathrm{kg} \mathrm{m}^{2}\right]\end{array}$ & $\begin{array}{l}\omega_{0} \\
{[\mathrm{~Hz}]}\end{array}$ & {$[\mathrm{rad} / \mathrm{s}]$} \\
\hline 1 & 2.05 & 60 & 12.0 & 27.0 & 72 & 79.4 & 499 \\
2 & 3.83 & 60 & 12.0 & 27.0 & 72 & 277 & 1740 \\
3 & 2.05 & 25 & 5.5 & 18.0 & 2.1 & 720 & 4520 \\
4 & 3.83 & 25 & 5.5 & 18.0 & 2.1 & 2510 & 15800 \\
\hline
\end{tabular}

with

$$
K=G \pi d^{4} / 32 \mathrm{~h},
$$

where $G$ is the shear modulus of the Thermelast $\left(62 \mathrm{kN} / \mathrm{mm}^{2}\right)$.

The moment of inertia of a cylindrical mass is given by

$$
I=\pi \varrho l r^{4} / 2 \text {. }
$$

With (20) and (21) the expression for the resonance frequency is:

$$
\omega_{0}=\left(d^{2} / 4 r^{2}\right) \sqrt{G / \varrho l h} .
$$

The temperature dependence of the resonance frequency resulting from (22) is:

$$
\frac{1}{\omega_{0}} \frac{d \omega_{0}}{d T}=\frac{3}{2} \alpha_{R}-\alpha_{M}+\frac{1}{2 G} \frac{d G}{d T},
$$

where $\alpha_{R}$ and $\alpha_{M}$ are the coefficients of linear expansion of the torsion rod and the torsion mass respectively.

With known $\alpha_{R}$ and $\alpha_{M}$ the last heat treatment of the torsion rod can be chosen such that the temperature dependence of the resonance frequency is less than $5 \cdot 10^{-6}{ }^{\circ} \mathrm{C}^{-1}[10]$. In our case the heat treatment is 0.5 hour at $650^{\circ} \mathrm{C}$.

The pendulum mass is machined from non-magnetic stainless steel (DIN 4401). The torsion-rod connections to the pendulum mass and the base plate bracket are hard solder connections made by high-frequency soldering. During soldering a tool is used for proper alignment of the parts. The cylindrical magnet is made of isotropic Alnico 260, manufacturer Magnetfabrik Bonn, Germany. The magnet is glued inside the pendulum mass with Loctite 601 . The bottom cover is machined after laser welding. The base plate bracket is screwed down to the base plate. In order to minimize the mechanical coupling between the pendulum mass and the base plate, the moment of inertia of the base plate is taken at least 50.000 times the moment of inertia of the pendulum mass. All the construction materials in the neighbourhood of the magnet, like the coil holders and supports, are made out of a fiber reinforced phenol formaldehyde. This material is used for its stiffness, its coefficient of expansion, which is close to that of the copper coils, and because of its non-conductivity. Conducting materials would increase the damping of the pendulum due to eddy-current effects.

The temperature control is established by circulating water from a thermostat through the double walled sample holder, which is made of glass.

The minimum distance from the side surface of the pendulum to the inner sample holder wall is governed by the requirement that the shear wave must almost be damped out when it reaches the wall. The distance at which the amplitude of a plane-shear wave is damped out by a factor of $1 / e$ is given by [11]:

$$
\delta=\frac{1}{\omega} \sqrt{\frac{2}{\varrho}\left(\frac{G^{2}+G^{\prime 2}}{\sqrt{G^{2}+G^{\prime 2}}-G^{\prime}}\right)} .
$$

For a Newtonian liquid with a viscosity of $0.1 \mathrm{~Pa} \mathrm{~s}$, a density of $10^{3} \mathrm{~kg} / \mathrm{m}^{3}$ and at a frequency of $80 \mathrm{~Hz}$, the amplitude of the shear wave is attenuated by a factor of 1000 over a distance of $4 \mathrm{~mm}$.

\subsection{The magnet-coil system}

The actual coil system is composed of six identical coils, rectangular shaped, with height $22.5 \mathrm{~mm}$, length $40 \mathrm{~mm}$, wire diameter $0.05 \mathrm{~mm}$ and 4000 turns each. The cylindrical magnet is diametrically magnetized. The relative positions of the coils and magnet are shown in figure 4. Except for the noise-suppression coils, this set-up is the same as used by Brouwer et al. [12].

The excitation current is sent through coils 1 and 2 (in parallel, with the same winding sense). The direction of the magnetic dipole is towards the edges of the measuring coils. This dipole direction has two advantages. First, the induction voltage is proportional to the angular velocity (see below) and second, the induction voltage is maximum for this position. The noise-suppression coils 5 and 6 are connected in series with the measuring coils 3 and 4 , respectively, but the winding sense of coils 5 and 6 is opposite to that of 3 and 4. By this arrangement the noise due to far fields is reduced to a minimum. The signals from the coil sets $3-5$ and $4-6$ are added and amplified. Because of the symmetrical arrangement of the coils the cross-talk between excitation coils and measuring coils is very small.

An estimation of the relation between the induction voltage and the angular amplitude can be made as follows. The magnetic induction $B$ of a dipole with dipole moment $p$, at a distance $x$ in the dipole direction, is given by

$$
B=\mu_{0} p / 2 \pi x^{3} \text {. }
$$

The induction voltage in the coils is equal to the change of magnetic flux per unit of time. The magnetic induction is maximum at one edge of the coil and minimum at the other edge. Therefore a small change in angular position of the magnet gives almost no change in the magnetic induction at the edges of the coils. The change of magnetic flux per unit time in the coil is then proportional to the angular velocity of the magnet. The induction-voltage amplitude $U_{0}$ can be expressed in the angular-displacement amplitude $\theta_{0}$ in good approximation by

$$
U_{0}=\sqrt{2} n \cdot B \cdot a \times \omega \theta_{0}=C_{2} \omega \theta_{0}
$$

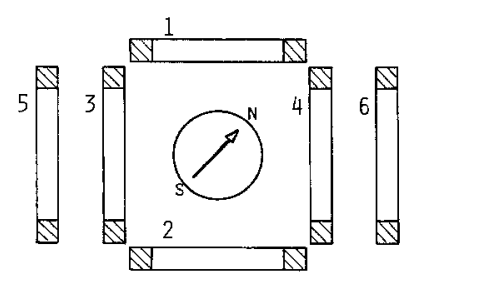

Fig. 4. Magnet-coil system. 1, 2: excitation coils; 3, 4: measuring coils; 5, 6: noise suppression coils. 
where $n$ is the number of turns per coil and $a$ is the height of the coil. By using (25) and (26) the apparatus constant is

$$
C_{2}=2 \sqrt{2} 10^{-7} \text { n a } p / x^{2} .
$$

Two different magnets have been used. A small one for the two higher frequencies and a bigger one for the two lower frequencies. The measured dipole moments are $0.1 \mathrm{Am}^{2}$ and $0.4 \mathrm{Am}^{2}$ respectively. With the values for $x=2.3 \cdot 10^{-2} \mathrm{~m}$, $a=2.25 \cdot 10^{-2} \mathrm{~m}$, and $n=4000$, the two different $C_{2}$ values are $2.3 \cdot 10^{-3} \mathrm{Vs} / \mathrm{rad}^{2}$ and $0.9 \cdot 10^{-2} \mathrm{Vs} / \mathrm{rad}^{2}$.

\section{Calibration}

The validity of eqs. $(15-18)$ has been investigated experimentally for all four torsion pendula. The reference measurements have been performed in air instead of in vacuum. A small correction (in the order of one fifth of the measured band-width in air) is therefore

Table 2. Pendulum properties (in vacuum) at $25^{\circ} \mathrm{C}$

\begin{tabular}{llll}
\hline $\begin{array}{l}\text { pendulum } \\
\text { number }\end{array}$ & $\begin{array}{l}\text { top angular } \\
\text { frequency } \\
{[\mathrm{rad} / \mathrm{s}]}\end{array}$ & $\begin{array}{l}\text { band-width } \\
{[\mathrm{rad} / \mathrm{s}]}\end{array}$ & $\begin{array}{l}\text { mechanical } \\
\text { quality } \\
Q\end{array}$ \\
\hline 1 & 498.266 & 0.016 & 31000 \\
2 & 1758.765 & 0.033 & 53000 \\
3 & 4500.44 & 0.10 & 45000 \\
4 & 15748.55 & 0.48 & 33000 \\
\hline
\end{tabular}

Table 3. Properties of the used Newtonian calibration liquids at $25.0 \pm 0.1^{\circ} \mathrm{C}$

\begin{tabular}{lll}
\hline sample & $\begin{array}{l}\text { density } \\
{\left[10^{3} \mathrm{~kg} / \mathrm{m}^{3}\right]}\end{array}$ & $\begin{array}{l}\text { viscosity } \\
{[\mathrm{m} \mathrm{Pa} \mathrm{s}]}\end{array}$ \\
\hline hexane & 0.653 & 0.294 \\
water & 0.997 & 0.890 \\
carbon tetra chloride & 1.581 & 0.905 \\
1-butanol & 0.807 & 2.60 \\
dibutylphthalate & 1.040 & 16.2 \\
di-(ethyl hexyl) phthalate & 0.978 & 58.0 \\
\hline
\end{tabular}

necessary. The properties of the pendula in vacuum are given in table 2. The temperature dependence of the resonance frequency is found to be less than $5 \cdot 10^{-6}$ ${ }^{\circ} \mathrm{C}^{-1}$ for all four pendula. The short-time (a few days) stability of the resonance frequencies is better than $5 \cdot 10^{-6}$. The long-time stability (a few months) is better than $2 \cdot 10^{-5}$.

With the help of six different Newtonian liquids the apparatus constants have been determined. The properties of the used liquids are given in table 3 .

The densities have been measured with the PAAR DMA 40 digital density meter. The viscosity values are literature values [13], except for the two phtalates, which have been measured with help of an Ubbelohde viscometer. All liquids used are of analytical grade.

From the measured band-widths (six values for each pendulum) the constants $A$ and $E$ have been calculated with a least square sum fit of (15). Also from the measured top frequencies the apparatus constants $A$ have been calculated from (16) by averaging.

The results are given in table 4, together with the theoretical values calculated with help of (17) and (18), by using the nominal pendulum dimensions (see table 1).

There are no significant differences between the $A$ values. However, for pendulum $4(2500 \mathrm{~Hz})$ the averaged $A$ value calculated from the top frequency is lower than the averaged $A$ value calculated from the band-width. Also the error is rather large. This may be due to the effect of an immobilized liquid layer in the surface roughness of the pendulum on the top frequency. A correction for this effect, which is proportional to the frequency itself, is used by Rouse et al. [14] and by Oosterbroek et al. [5]. An immobilized layer of $0.2 \mu \mathrm{m}$ can explain the differences between the two $A$ values.

The measured $E$ values are about $30 \%$ higher than the theoretical values. Schrag et al. [6] found an experimental value that was $50 \%$ higher than the theoretical one. The errors in the measured $E$ values are large, but

Table 4. Apparatus constants

\begin{tabular}{llllll}
\hline $\begin{array}{l}\text { pendulum } \\
\text { number }\end{array}$ & $\begin{array}{l}A \text { theory (17) } \\
{\left[\mathrm{kg} / \mathrm{m}^{2}\right]}\end{array}$ & $\begin{array}{l}A \text { measured (15) } \\
{\left[\mathrm{kg} / \mathrm{m}^{2}\right]}\end{array}$ & $\begin{array}{l}A \text { measured }(16) \\
{\left[\mathrm{kg} / \mathrm{m}^{2}\right]}\end{array}$ & $\begin{array}{l}E \text { theory (18) } \\
{\left[\mathrm{m}^{-1}\right]}\end{array}$ & $\begin{array}{l}E \text { measured (15) } \\
{\left[\mathrm{m}^{-1}\right]}\end{array}$ \\
\hline 1 & $40 \pm 1$ & $39.3 \pm 0.05$ & $39.3 \pm 0.3$ & $102 \pm 2$ & $140 \pm 20$ \\
2 & $40 \pm 1$ & $39.3 \pm 0.05$ & $39.2 \pm 0.3$ & $102 \pm 2$ & $130 \pm 20$ \\
3 & $19.3 \pm 0.5$ & $19.0 \pm 0.1$ & $18.8 \pm 0.2$ & $235 \pm 5$ & $270 \pm 40$ \\
4 & $19.3 \pm 0.5$ & $19.0 \pm 0.1$ & $18.2 \pm 0.8$ & $235 \pm 5$ & $270 \pm 40$ \\
\hline
\end{tabular}

i) The errors in the theoretical $A$ and $E$ values follow from the inaccuracy in pendulum dimensions and pendulum moment of inertia.

ii) The errors in the measured $A$ and $E$ values are standard-deviation values following from the curve-fit calculations and averagings. 
the influence on the accuracy of $X_{\mathrm{pl}}$ is small because the value of the correction term $E G^{\prime \prime} / \omega$ in (15) is at most $5 \%$ of the value of $X_{\mathrm{pl}}$.

\section{Measurement of a viscoelastic polymer solution}

A solution of polystyrene in dibutylphthalate is used as a demonstration of a measurement of a viscoelastic liquid. The polystyrene, with molecular weight of 470.000, was obtained from Waters Ass., Milford. The concentration is $1.8 \%$ by weight. The dibutylphthalate, with purity $>99 \%$, was obtained from Merck-Schuchardt, Hohenbrunn.

The relative (with respect to dibutylphtalate) steadystate viscosity of the solution is 3.50 , measured with the Contraves Low Shear 30.

The dynamic moduli of the solution have been measured with the four torsion pendula and with the nickel-tube apparatus, developed in our laboratory [5].

The apparatus constants used for the torsion-pendulum measurements are for the two lower frequencies $A=39.3 \mathrm{~kg} / \mathrm{m}^{2}$ and $E=130 \mathrm{~m}^{-1}$ and for the two higher frequencies $A=19.0 \mathrm{~kg} / \mathrm{m}^{2}$ and $E=270 \mathrm{~m}^{-1}$. Only one iteration is needed to calculate the $X_{\mathrm{pl}}$ and $R_{\mathrm{pl}}$ within $0.5 \%$.

The frequency range of the nickel-tube apparatus is extended with respect to that reported by Oosterbroek [5]. The first mode of the tube at $3.7 \mathrm{kHz}$ is incorporated as well as a few overtones above $167 \mathrm{kHz}$. The total used range is now $3.7-234 \mathrm{kHz}$. The results of the pendulum and nickel-tube measurements are given in figure 5 .

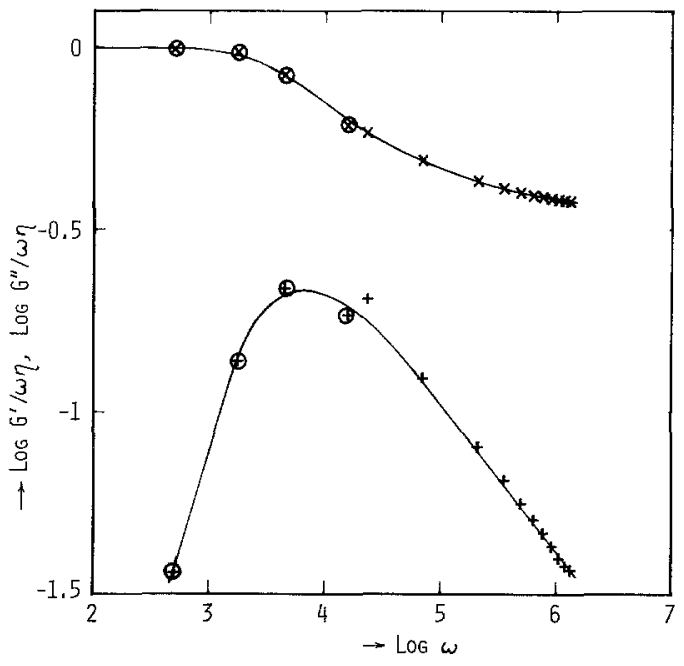

Fig. 5. Relative dynamic moduli of $1.8 \% \mathrm{w} / \mathrm{w}$ polystyrene 470,000 in dibutylphthalate as a function of frequency; $x$ : $\log \left(G^{\prime} / \omega \eta\right),+: \log \left(G^{\prime \prime} / \omega \eta\right)$, the encircled points have been measured with the torsion pendula.
The value of $G^{\prime \prime} / \omega \eta$ at $3.7 \mathrm{kHz}$ deviates (about $5 \%$ ) from the tentative drawn curve. This is probably due to the fact that the fundamental resonance of the nickel tube has no nodal point at the attachment points of the tube.

\section{Determination of maximum rate of shear}

The apparatus constant $C_{2}$ is used to calculate the maximum rate of shear in the sample. The estimation of the value of $C_{2}$ is checked by measuring the torsion amplitude as function of the induction-voltage amplitude. A small thin mirror is glued onto the cylindrical surface of the pendulum mass. A laser beam is reflected by this mirror. The length of the line that the reflected beam makes on a screen at $6 \mathrm{~m}$ distance from the pendulum is then measured. The angular amplitude follows from this length. The results for $79 \mathrm{~Hz}$ and the $716 \mathrm{~Hz}$ pendulum are given in figure 6.

The values for $C_{2}$ following from figure 6 are $2.4 \cdot 10^{-3} \mathrm{Vs} / \mathrm{rad}^{2}$ and $1.0 \cdot 10^{-2} \mathrm{Vs} / \mathrm{rad}^{2}$ for the $716 \mathrm{~Hz}$ and $79 \mathrm{~Hz}$ pendulum respectively.

When the approximations are taken into account, the calculated values $\left(2.3 \cdot 10^{-3} \mathrm{Vs} / \mathrm{rad}^{2}\right.$ and $0.9 \cdot 10^{-2} \mathrm{Vs} /$ $\mathrm{rad}^{2}$ respectively) and the measured values of $C_{2}$ are in reasonable agreement. Therefore (27) can serve for design purposes.

For linear viscoelastic fluids the maximum rate of shear for plane waves is given by

$$
\dot{\gamma}_{\max }=\omega r \theta_{0} \sqrt{\frac{\omega^{2} \varrho}{\sqrt{G^{\prime 2}+G^{\prime 2}}}}=\frac{U_{0} r}{C_{2}} \sqrt{\frac{\omega^{2} \varrho}{\sqrt{G^{\prime 2}+G^{\prime 2}}}} .
$$

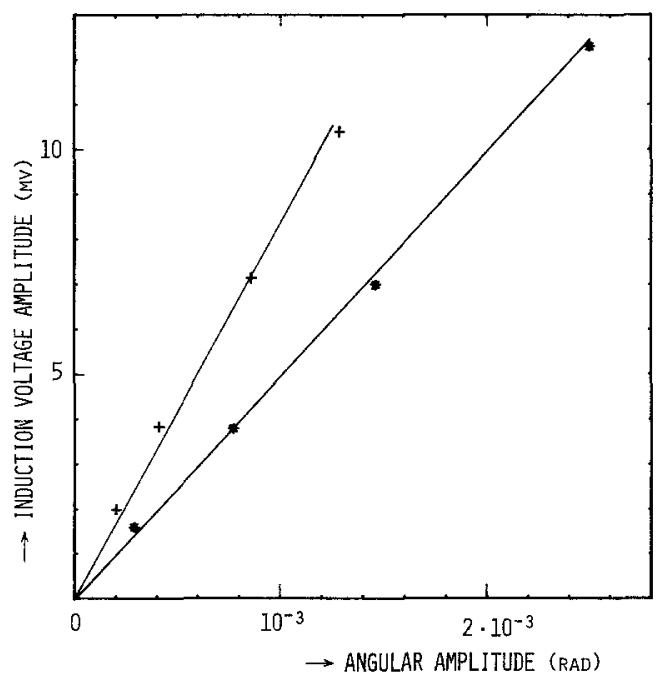

Fig. 6. Angular amplitude as a function of the inductionvoltage amplitude; +: $706 \mathrm{~Hz}$ pendulum, *: $79 \mathrm{~Hz}$ pendulum. 
Table 5. Comparison of different instruments

\begin{tabular}{|c|c|c|c|c|}
\hline & Sittel [7] & Konno [8], Benzing [3] & Schrag [6], Johnson [9] & this paper \\
\hline detection system & optical & electromagnetic & optical & electromagnetic \\
\hline geometry & hollow disk & plate & five stacked cylinders & single cylinder \\
\hline frequency range & $200-2300 \mathrm{~Hz}$ & $100-300 \mathrm{~Hz}$ & $100-6000 \mathrm{~Hz}$ & $80-2500 \mathrm{~Hz}$ \\
\hline $\begin{array}{l}\text { relative temp. coeff. } \\
\text { res. freq. }\end{array}$ & $-240 \cdot 10^{-6}{ }^{\circ} \mathrm{C}^{-1}$ & $?$ & $-290 \cdot 10^{-6}{ }^{\circ} \mathrm{C}^{-1}$ & less than $5 \cdot 10^{-6}{ }^{\circ} \mathrm{C}^{-1}$ \\
\hline sample size & $30 \cdot 10^{-6} \mathrm{~m}^{3}$ & $\sim 50 \cdot 10^{-6} \mathrm{~m}^{3}$ & $42 \cdot 10^{-6} \mathrm{~m}^{3}$ & $\begin{array}{l}2 \text { lower frequencies } \\
15 \cdot 10^{-6} \mathrm{~m}^{3} \text {, } \\
2 \text { higher frequencies } \\
5 \cdot 10^{-6} \mathrm{~m}^{3}\end{array}$ \\
\hline viscosity range & $5 \cdot 10^{-4}-5 \cdot 10^{-2} \mathrm{~Pa} \mathrm{~s}$ & $?$ & $5 \cdot 10^{-4}-5 \cdot 10^{-2} \mathrm{~Pa} \mathrm{~s}$ & $3 \cdot 10^{-4}-6 \cdot 10^{-2} \mathrm{~Pa} \mathrm{~s}$ \\
\hline accuracy in $\eta$ & up to $10 \%$ & $?$ & $1 \%$ & $1 \%$ \\
\hline method & $\begin{array}{l}\text { free oscillation, } \\
\text { frequency and decay } \\
\text { measurement }\end{array}$ & $\begin{array}{l}\text { forced oscillation, } \\
\text { at resonance frequency, } \\
\text { phase measurement }\end{array}$ & $\begin{array}{l}\text { forced oscillation, } \\
\text { measurement of } \\
\text { phase-frequency curve }\end{array}$ & $\begin{array}{l}\text { forced oscillation, } \\
\text { measurement of } \\
\text { amplitude-frequency } \\
\text { curve }\end{array}$ \\
\hline
\end{tabular}

The calibration measurements have been performed at a constant induction voltage amplitude per frequency. The shear rates at which these measurements have been performed are about $150 \mathrm{~s}^{-1}$ and $10 \mathrm{~s}^{-1}$ for the lowest and highest viscosity, respectively, chosen to be the same for each frequency. Thus the angular amplitudes used are $5 \cdot 10^{-4} \mathrm{rad}$ to $5 \cdot 10^{-6} \mathrm{rad}$ for the lowest and highest frequency respectively.

The lowest possible shear rates at which the signalnoise ratio is still good enough for a measurement is about ten times lower than used during the calibration measurements. The maximum possible shear rate limited by the available excitation power is about five times larger than that indicated above. The dynamic range of about 50 enables a check on the linearity of the viscoelastic properties.

\section{Discussion}

In the previous sections it is demonstrated that the presented torsion pendula operate quite well according to the idealized theoretical model when they are surrounded with low-viscous $\left(\eta^{\prime}<10^{-1} \mathrm{~Pa} \mathrm{~s}\right)$ fluids. The high mechanical stability of the set-up and the large mechanical quality $(Q$ more than 30,000) facilitate accurate measurements. The lower limit of the elastic effect $G_{\min }^{\prime}$, which can be detected significantly in the mentioned viscosity region, is given by the relation $G_{\min }^{\prime} / G^{\prime \prime} \simeq 0.03$. The $G_{\min }^{\prime}$ value is defined as $G_{\min }^{\prime}$ $\simeq 3 \Delta G_{\min }^{\prime}$, where $A G_{\min }^{\prime}$ is the standard deviation.

The variability of the maximum rate of shear with a factor of about 50 enables checks on the linearity of the viscoelastic behaviour. A comparison with other apparatus operating in the same frequency range is given in table 5 .

A few differences can be deduced. An advantage of the present design is the temperature independence of the resonator frequency. This avoids extreme temperature control. The detection system is very simple compared to that of the multiple lumped resonator of Schrag and Johnson $[6,9]$. The smaller sample quantity compared to the other instruments may be, advantageous in case of liquids that are expensive or scant.

Research programs on low-viscous viscoelastic systems often require a large number of measurements, where structure parameters determining the viscoelastic behaviour are varied systematically. In these cases the number of apparatus needed for the measurements of $G^{*}$ over a large frequency range and the length of measurement times are limiting factors for the character and volume of a program fixed in term. The development of a multiple torsion pendulum in a selfoscillating circuit is a promising next step to relaxing these limiting factors.

\section{Acknowledgements}

The authors wish to thank Mr. J. H. Westendorp for his excellent machine work and Mr. E. G. Altena and Mr. G. J. Beukema for their technical support.

\section{References}

1. Ferry JD, Viscoelastic properties of polymers, John Wiley \& Sons (New York 1969) 
2. Oosterbroek M, Mellema J, Lopulissa JS (1981) J Colloid Interface Sci 84:27

3. Benzing DW, Russel WB (1981) J Colloid Interface Sci 83: 178

4. Chmiel H, Walitza E, On the rheology of blood and synovial fluids, Research Studies Press (Chichester 1980)

5. Oosterbroek M, Waterman HA, Wiseall SS, Altena EG, Mellema J, Kip GAM (1980) Rheol Acta 19:497

6. Schrag JL, Johnson MJ (1971) Rev Scient Instr 42:224

7. Sittel K, Prince E et al. (1954) J Appl Phys 25:1312

8. Konno A, Makino S, Kaneko M (1968) Jap J Appl Phys $7: 89$

9. Johnson RM, Ph.D. Thesis, University of Wisconsin, 1970

10. Material specification F001 Thermelast, Vacuumschmelze GmbH, Hanau, Germany

11. Mason WP, Physical Acoustics, Vol. I-A, Chap. 1, Acad Press (New York 1964)
12. Brouwer AJ, Groenenbaum-Eggelaar C (1967) Acta Met 15:1597

13. Weast RC, Handbook of Chemistry and Physics, 51th ed., CRC Press (Cleveland, Ohio 1970)

14. Rouse PE Jr, Bailey ED, Minkin JA (1950) Proc A.P.I. $30 \mathrm{M}: 54$

(Received April 22, 1983)

Authors' address:

Ir. C. Blom, Dr. J. Mellema

Department of Applied Physics

Twente University of Technology

P. O. Box 217

NL-7500 AE Enschede 